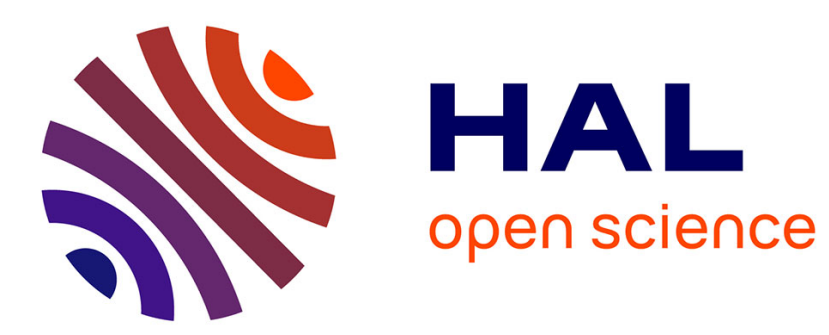

\title{
Form factor of rounded objects: the sections method \\ Bernard Croset
}

\section{To cite this version:}

Bernard Croset. Form factor of rounded objects: the sections method. Journal of Applied Crystallography, 2018, 51 (4), pp.51 - 51. 10.1107/S1600576718007239 . hal-01830520

\section{HAL Id: hal-01830520 \\ https://hal.sorbonne-universite.fr/hal-01830520}

Submitted on 5 Jul 2018

HAL is a multi-disciplinary open access archive for the deposit and dissemination of scientific research documents, whether they are published or not. The documents may come from teaching and research institutions in France or abroad, or from public or private research centers.
L'archive ouverte pluridisciplinaire HAL, est destinée au dépôt et à la diffusion de documents scientifiques de niveau recherche, publiés ou non, émanant des établissements d'enseignement et de recherche français ou étrangers, des laboratoires publics ou privés. 


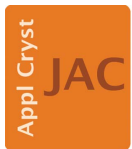

JOURNAL OF

APPLIED

CRYSTALLOGRAPHY

ISSN 1600-5767

Received 5 March 2018

Accepted 14 May 2018

Edited by V. T. Forsyth, Institut Laue-Langevin, France, and Keele University, UK

Keywords: form factors; analytical methods; fourfold truncated sphere; surface singularities.

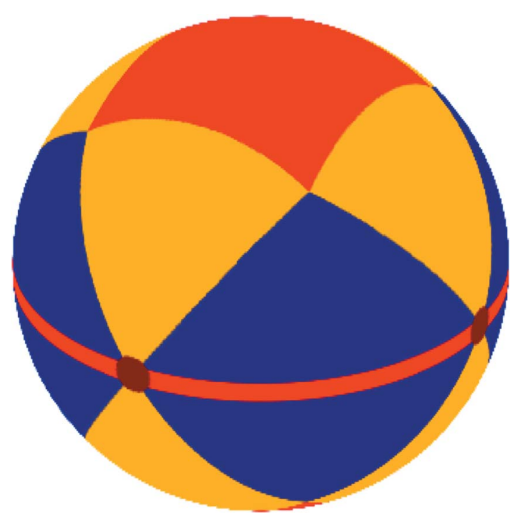

(C) 2018 International Union of Crystallography

\section{Form factor of rounded objects: the sections method}

\author{
Bernard Croset*
}

Institut des NanoSciences de Paris, CNRS-UMR 7588, Sorbonne Université, F-75005 Paris, France. *Correspondence e-mail: bernard.croset@insp.jussieu.fr

An analytical method, the sections method, is developed to build a close link between the singularities of the surface of a body and the asymptotic behaviour of its amplitude form factor at large scattering vector, q. In contrast with a sphere, for which the asymptotic behaviour is in $q^{-2}$, surface singularities lead to both narrow regions, for which the amplitude form factor exhibits trailing behaviour, and extended regions, for which it exhibits a rapid decrease. A numerical study of a simple example, the fourfold truncated sphere, illustrates the usefulness of these analytical predictions.

\section{Introduction}

As pointed out previously, nanoparticles and their shapes play an ever increasing role in condensed matter physics (Balmes et al., 2016; Goubet et al., 2013; Gruner et al., 2008; Langille et al., 2012; Murphy et al., 2005; Xia et al., 2009; Xie et al., 2012; Yamada et al., 2011; Zhang et al., 2012). X-ray scattering techniques are an important tool to study and follow the fabrication of nanoparticles and their self-assembly (Cho et al., 2005; Geuchies et al., 2016; Goubet et al., 2016; Jones et al., 2010; Kalesaki et al., 2014; Lee et al., 2011; Renaud et al., 2009). Owing to rapid progress in the brightness of X-ray sources and in phase-retrieval algorithms, coherent small-angle X-ray scattering or scattering around Bragg peaks are appropriate tools for the determination of nanoparticle shapes (Diaz et al., 2009; Favre-Nicolin et al., 2009; Labat et al., 2015; Miao et al., 2015; Öztürk et al., 2017).

As pointed out by Senesi \& Lee (2015), the calculation of the form factor of the particles is of major importance in the context of any scattering technique. The use of Green's theorem and surface triangulation approaches (Georg \& Tausch, 1994) leads to very efficient numerical methods for particles having 'smooth' surfaces (Chourou et al., 2013). Besides these numerical techniques and phase-retrieval algorithms, the ability to predict analytically the main characteristics of the amplitude form factor for a given shape of a scattering body may be quite useful. In a previous paper (Croset, 2017), we established a compact formula for the amplitude form factor of any polyhedron whatever its convexity, and we showed that it presents asymptotic behaviour in $q^{-3}$ for a generic direction of the scattering vector $\mathbf{q}$, in $q^{-2}$ for directions perpendicular to an edge and in $q^{-1}$ for directions normal to a face. Our purpose in this paper is to address the generalization of such a result for more rounded bodies. We will show that the dependence of the asymptotic behaviour of the amplitude form factor on the scattering vector orientation can be established analytically using an expression for the amplitude form factor already proposed by 
Patterson (1939). Finally, a numerical application to a fourfold truncated sphere demonstrates the physical usefulness of this analytical approach.

\section{The sections method}

\subsection{The Patterson result}

We want to calculate the amplitude form factor of a given body, $\mathscr{B}$. We have

$$
F(\mathbf{q})=\iiint_{\mathscr{B}} \exp (-i \mathbf{q} \cdot \mathbf{r}) \mathrm{d}^{3} \mathbf{r} .
$$

Choosing as our Cartesian coordinate system, $(x, y, z)$, a system having its $z$ axis parallel to $\mathbf{q}, F$ is written as

$$
F(\mathbf{q})=\int\left(\iint_{\mathscr{S}(h)} \mathrm{d} x \mathrm{~d} y\right) \exp (-i q h) \mathrm{d} h=\int A(h) \exp (-i q h) \mathrm{d} h,
$$

where $\mathscr{S}(h)$ is the section of the body by the plane $\mathscr{P}$, normal to $\mathbf{q}$ at $z=h$, and $A(h)$ is its area. This result, first established by A. L. Patterson in 1939, is well known in crystallography. We will show in this paper that it is of major interest, since it allows us to discuss the asymptotic behaviour of $F(\mathbf{q})$ at large $q$ and its dependence on the orientation of the scattering vector. Indeed, whereas the Fourier transform of $A(h)$ cannot, in general, be calculated analytically, the singularities of $A(h)$ can be identified and described analytically. The theory of distributions shows that there is a direct link between the singularities of a function and the asymptotic behaviour of its Fourier transform (Guelfand \& Chilov, 1962). Therefore, equation (2) is a clue to discussing the asymptotic behaviour of $F(\mathbf{q})$.

\subsection{Classification of the singularities of $A(h)$}

Since the scattering body has a finite support, $A(h)$ always exhibits two singularities at $h=h_{\mathrm{i}}$, the first contact of $\mathscr{P}$ with the body, and $h=h_{\mathrm{o}}$, the last contact of $\mathscr{P}$ with the body. We will call these two singularities 'entry' and 'exit', respectively. When the external surface of the body exhibits singularities truncation, edges, apices etc. - the contact of $\mathscr{P}$ with these singularities leads to singularities in $A(h)$ in the interval $h_{\mathrm{i}} \geq$ $h \geq h_{\mathrm{o}}$. For each singularity of $A(h)$ in $h_{n}$, the main point is to determine the characteristic exponent of the singularity, $\lambda_{n}$. Defining $\varepsilon=\left(h-h_{n}\right), \lambda_{n}$ corresponds to the power of the leading term of the difference between the series developments of $A(h)$ for positive, $A_{+}(|\varepsilon|)$, and negative, $A_{-}(-|\varepsilon|)$, values of $\varepsilon$, i.e.

$$
S(|\varepsilon|)=\left|A_{+}(|\varepsilon|)-A_{-}(-|\varepsilon|)\right|=D|\varepsilon|^{\lambda_{n}}+\mathcal{O}\left(|\varepsilon|^{\lambda_{n}+1}\right) .
$$

\subsection{Asymptotic behaviour of the amplitude form factor}

From the theory of distributions (see e.g. Guelfand \& Chilov, 1962), it is well known that a singularity for $A(h)$ of exponent $\lambda$ and amplitude $D$ leads to an asymptotic term for the Fourier transform of $A$ which can be written $D q^{-(\lambda+1)}+\mathcal{O}\left[q^{-(\lambda+2)}\right]$. Using this result, to determine the asymptotic behaviour of $F(\mathbf{q})$ in a given direction we just have to identify the different discontinuities of $A(h)$ in this direction and determine their characteristic exponent, $\lambda_{n}$. Note that the asymptotic behaviour will be determined by the smallest value of $\lambda_{n}$. We will call this the 'rule of dominant singularities'. Therefore, to study the dependence of $F(\mathbf{q})$ on the $\mathbf{q}$ direction, we have to study the singularities of $A(h)$ for every direction and apply the rule of dominant singularities. This constitutes the 'sections method'. The next section will be devoted to an illustrative example, the case of the tetrahedron for which Croset (2017) provides an exact formula. In $\$ 4$ we will study the more common discontinuities and the associated asymptotic terms. Finally, in $\S 5$ we will show that this study of asymptotic behaviour can be used to predict the contrast in the variation of the amplitude form factor with $\mathbf{q}$ direction at rather small values of $q$.

\section{Principle of the method: the tetrahedron example}

Even though the compact formula established for the amplitude form factor of a polyhedron gives an exact analytical result for the tetrahedron amplitude form factor, we will study this case by the sections method in order to demonstrate the principles of the method and give illustrative details of its application.

\subsection{Generic direction}

For a generic direction, entry and exit occur by contact with an apex for $h=h_{\mathrm{i}}$ and $h=h_{\mathrm{o}}$, and the other two apices are met consecutively for $h=h_{1}$ and $h=h_{2}$. In such a case, the section is a triangle $T_{\mathrm{i}}$ for $h_{\mathrm{i}} \geq h \geq h_{1}$ and a triangle $T_{\mathrm{o}}$ for $h_{2} \geq h \geq h_{\mathrm{o}}$. These sections vary homothetically with $\varepsilon_{\mathrm{i}}=h_{\mathrm{i}}-h$ and $\varepsilon_{\mathrm{o}}=h$ $-h_{\mathrm{o}}$, leading to $\lambda_{\mathrm{i}}=\lambda_{\mathrm{o}}=2$. For $h_{1} \geq h \geq h_{2}$, the section is a quadrilateral, $Q$. The difference between $Q$ and $T_{\mathrm{i}}$ (or $T_{\mathrm{o}}$ ) is a triangle $T_{1}$ (or $T_{2}$ ) varying homothetically with $\varepsilon_{1}=h_{1}-h$ (or $\varepsilon_{2}=h-h_{2}$ ), leading to $\lambda_{1}=\lambda_{2}=2$. Fig. 1 illustrates this point. Therefore, for $\mathbf{q}$ in a generic direction, the asymptotic behaviour of $F(\mathbf{q})$ is in $q^{-3}$. We retrieve the result established directly using the formula of Croset (2017). Note that we will

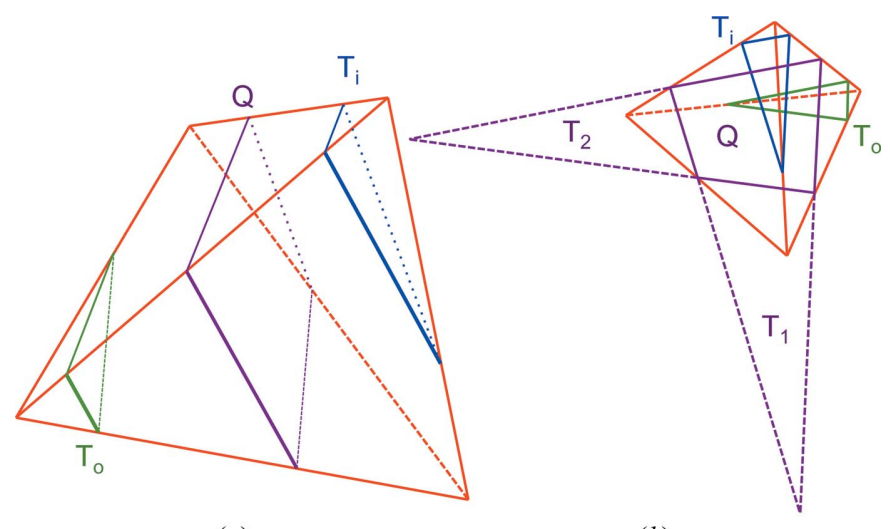

(a)

(b)

Figure 1

Sections of the tetrahedron in a generic direction. (a) Perspective view and $(b)$ a view from the normal to $\mathscr{P}$. The tetrahedron edges are drawn in red. The different sections are drawn in blue, purple and green. 
meet this behaviour several times in this article: a positive contribution, here the triangles $T_{\mathrm{i}}$ and $T_{\mathrm{o}}$, or a negative contribution, here the triangles $T_{1}$ and $T_{2}$, of a given shape to the section singularity leading to the same $\lambda$ and the same asymptotic behaviour.

\section{2. q perpendicular to an edge}

If $\mathbf{q}$ is perpendicular to an edge, we have $h_{\mathrm{i}}=h_{1}$ or $h_{1}=h_{2}$ or $h_{2}=h_{\mathrm{o}}$. Let us study the case when $h_{\mathrm{i}}=h_{1}$. For $h_{1} \geq \mathrm{h} \geq h_{2}$, the section is a trapezoid of constant angles: its bases are parallel to the edge and have a length equal to the edge length at first order in $\varepsilon$, while its height is proportional to $\varepsilon$. Therefore, for the singularity in $h=h_{1}, \lambda=1$. For $h_{2} \geq h \geq h_{\mathrm{o}}$, we retrieve the triangle $T_{\mathrm{o}}$ and the singularities in $h=h_{2}$ and $h=h_{\mathrm{o}}$ are unchanged with $\lambda=2$. Thus, in this case, following the rule of dominant singularities the asymptotic behaviour of $F(\mathbf{q})$ is in $q^{-2}$. By symmetry, this asymptotic behaviour is the same for the case $h_{2}=h_{\mathrm{o}}$. For the case when $h_{1}=h_{2}, T_{\mathrm{i}}$ and $T_{\mathrm{o}}$ are similar triangles and we go directly from $T_{\mathrm{i}}$ to $T_{\mathrm{o}}$ in $h=h_{1}$. The curve $A(h)$ therefore consists of two parabolic arcs of equation $a_{\mathrm{i}}\left(h-h_{\mathrm{i}}\right)^{2}$ and $a_{\mathrm{o}}\left(h-h_{\mathrm{o}}\right)^{2}$, and presents a slope discontinuity in $h=h_{1}=h_{2}$ leading to $\lambda=1$, while the two singularities in $h=$ $h_{\mathrm{i}}$ and $h=h_{\mathrm{o}}$ remain unchanged. Once again, following the rule of dominant singularities the asymptotic behaviour of $F(\mathbf{q})$ is in $q^{-2}$. Thus, in all three cases we retrieve an asymptotic behaviour of $F(\mathbf{q})$ in $q^{-2}$, which corresponds to the result established directly using the formula of Croset (2017).

\section{3. q normal to a face}

If $\mathbf{q}$ is normal to a face, we have $h_{\mathrm{i}}=h_{1}=h_{2}$ or $h_{1}=h_{2}=h_{\mathrm{o}}$. In both cases, we have a discontinuity of the section area when touching the face leading to $\lambda=0$ and an asymptotic behaviour of $F(\mathbf{q})$ in $q^{-1}$, which corresponds to the result established directly using the formula of Croset (2017).

\section{Usual discontinuities for rounded bodies}

In order to find and classify the different singularities of $A(h)$, we first have to identify the singularities of the body surface and then study the orientation of $\mathbf{q}$ with respect to these shape singularities. We will not try to make an exhaustive study of the singularities of surfaces but we will explore the ones which seem to be more common in physical problems.

Let us first discuss the main singularities of the body surface.

(i) The scattering body may be fully spheroidal. In this case the surface does not present any singularity and two main radii of curvature can be defined at each point.

(ii) The scattering body may be ruled, as are revolution cylinders and revolution cones.

(iii) The scattering body may present truncations.

(a) In the case of a single truncation (i.e. a face), the perimeter of this truncation is generally an edge, i.e. a continuous set of points on the surface where there are two tangent planes. (b) In the case of two secant truncations, there is a straight edge. This straight edge has two extremities which are apices.

For a given surface, $A(h)$ will present singularities not only at the entry and exit points, but also each time the intersecting plane $\mathscr{P}$ encounters a singularity of the surface. The characteristic coefficient $\lambda$ depends on both the nature of the singularity and the orientation of $\mathbf{q}$ with respect to this singularity. The following subsections review the main possibilities. Note that the singularities we will discuss appear in the same form for convex and concave objects, since local convexity controls only the sign of $S(\varepsilon)$ for these singularities. Nevertheless, concave objects may present points for which the two main radii of curvature have different signs (saddle points, for example). We will not discuss such cases in this paper.

\section{1. $\lambda=0$ : surface contact with a truncation}

If $\mathbf{q}$ is normal to a face, $\mathscr{F}$, of area $A_{\mathscr{F}}$, there is a value of $h$ where $\mathscr{F}$ is in $\mathscr{P}$ and for which $A(h)$ presents a discontinuity of amplitude $A_{\mathscr{F}}$, leading to an asymptotic behaviour in $A_{\mathscr{F}} q^{-1}$. This behaviour corresponds to the well known truncation rods which appear for surfaces of semi-infinite crystals in grazingincidence X-ray scattering (GIXS) (Robinson, 1986), for faceted vicinal faces in GIXS (Coati et al., 2005) and for faceted aggregates in grazing-incidence small-angle X-ray scattering (Renaud et al., 2009).

\section{2. $\lambda=1 / 2$ : line contact with a generatrix}

Let us begin with the simplest example, the right revolution cylinder of radius $R$ and height $H$ (see Fig. 2). Direct calculation of the amplitude form factor gives

$$
F(\mathbf{q})=2 \pi R^{2} H \frac{J_{1}\left(q_{\|} R\right)}{q_{\|} R} \frac{\sin \left(q_{z} H / 2\right)}{q_{z} H / 2},
$$

where $q_{\|}$and $q_{z}$ are the components of $\mathbf{q}$ normal and parallel to the cylinder axis, respectively, and $J_{1}$ is the Bessel function of the first kind of order 1 [Fournet, 1951; Guinier \& Fournet, 1955; and also Lazzari (2002), who presents a large catalogue of form factors]. For such a cylinder, if $\mathbf{q}$ is normal to the axis, then for $R=h_{\mathrm{i}} \geq h \geq h_{\mathrm{o}}=-R$ the section is a rectangle of length $H$ and width $2\left(R^{2}-h^{2}\right)^{1 / 2}$. The first term of the series

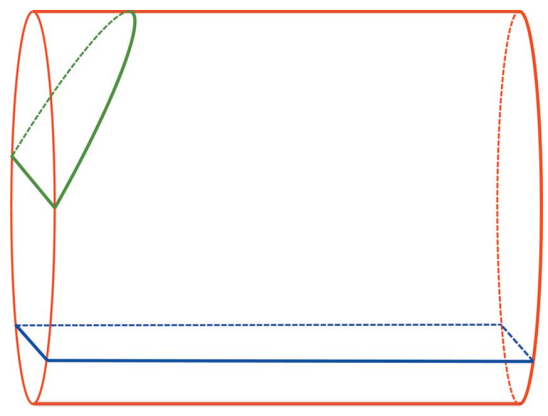

Figure 2

Sections of the revolution cylinder. Red denotes the surface of the cylinder, blue the section by a plane parallel to the generatrices and green a section in a generic direction. 
development of the section area discontinuities at the two critical values $h=h_{\mathrm{i}}$ and $h=h_{\mathrm{o}}$ is written $S(\varepsilon)=2 H(2 R \varepsilon)^{1 / 2}$, leading to $\lambda=1 / 2$ for the two singularities and to an asymptotic behaviour in $2 H(2 R)^{1 / 2} q^{-3 / 2}$ for $F(\mathbf{q})$. Since $J_{1}(x) / x$ decreases asymptotically as $x^{-3 / 2}$, the asymptotic behaviour found using the sections method is in full agreement with that predicted by the direct calculation of $F(\mathbf{q})$.

The sections method has the great advantage of predicting asymptotic behaviour for more complex cases for which the amplitude form factor cannot be calculated analytically. Firstly, let us consider a general cylinder of which the directrix is a planar curve without apices, i.e. having a radius of curvature $R(\mathbf{t})$ defined at any point $\mathbf{t}$. For $\mathbf{q}$ normal to the generatrices' direction, we will denote as $\mathbf{t}_{\mathrm{i}}$ the point of first contact of the plane $\mathscr{P}$ with the cylinder corresponding to $h=$ $h_{\mathrm{i}}$. For $h_{\mathrm{i}} \lesssim h$, the section is a rectangle of height $H\left(\mathbf{t}_{\mathrm{i}}\right)$ and width $2\left[R\left(\mathbf{t}_{\mathbf{i}}\right)^{2}-h^{2}\right]^{1 / 2}$. We retrieve for the first term of the series development of the section area discontinuity $S(\varepsilon)=$ $2 H\left(\mathbf{t}_{\mathbf{i}}\right)\left[2 R\left(\mathbf{t}_{\mathbf{i}}\right) \varepsilon\right]^{1 / 2}$, leading to $\lambda=1 / 2$. Note that the precise shape and orientation of the two cylinder bases which control the variation of $H\left(\mathbf{t}_{\mathbf{i}}\right)$ with $\mathbf{t}_{\mathrm{i}}$ do not play a role in the value of $\lambda$ since they do not affect the degree of the first term of the series development of $S(\varepsilon)$. Secondly, it is easy to be persuaded that the result established for a general cylinder is retained when $\mathscr{P}$ touches the surface of a cone by a generatrix (see Appendix $A$ for detailed calculations).

\section{3. $\lambda=1$ : contact with a spheroid and contact with a straight} edge

4.3.1. Point contact with a sphere or a spheroid. Let us first examine the case of a sphere of radius $R$. The amplitude form factor can be calculated directly to obtain the well known result

$$
F(\mathbf{q})=4 \pi[\sin (q R)-q R \cos (q R)] q^{-3},
$$

which has an asymptotic behaviour in $4 \pi R \cos (q R) q^{-2}$. Now, using the sections method, for any direction of $\mathbf{q}$ and for $R=$ $h_{\mathrm{i}} \geq h \geq h_{\mathrm{o}}=-R$ the section is a circle of radius $r=\left(R^{2}-\right.$ $\left.h^{2}\right)^{1 / 2}$, leading to $A(h)=\pi\left(R^{2}-h^{2}\right)$. The first term of the series development of the area discontinuity is given by $S(\varepsilon)=2 \pi R \varepsilon$ for both the entry singularity and the exit singularity. For both cases, we have $\lambda=1$ and an asymptotic behaviour in $2 \pi R q^{-2}$ for the amplitude form factor, as predicted by the direct formula.

The sections method allows us to generalize this result to a point contact with a spheroidal part of a surface. In this case, at the point contact, the surface possesses two main radii of curvature, $R_{1}$ and $R_{2}$. To first order, the section is an ellipse of area $S(\varepsilon)=2 \pi\left(R_{1} R_{2}\right)^{1 / 2} \varepsilon$ and we get an asymptotic behaviour in $2 \pi\left(R_{1} R_{2}\right)^{1 / 2} q^{-2}$.

4.3.2. Line contact with a straight edge, i.e. with the intersection of two planar truncations. Let us study the case of contact with a straight edge. We have already met this case in the study of the tetrahedron, and the exact formula established by Croset (2017) predicts $\lambda=1$ for $\mathbf{q}$ perpendicular to an edge for any polyhedron. The sections method allows us to generalize this result to any straight edge. Let us examine the case of a surface with two planar truncations, $\mathscr{T}_{1}$ and $\mathscr{T}_{2}$, secant along an edge $\mathbf{A B}$. We will show that $A(h)$ possesses a singularity with $\lambda=1$ when $\mathbf{q}$ is perpendicular to $\mathbf{A B}$. Let us denote as $h_{\mathrm{c}}$ the value of $h$ for which $\mathbf{A B}$ is in $\mathscr{P}$. Three cases occur, two of them being equivalent: first, $\mathscr{P}$ intercepts neither $\mathscr{T}_{1}$ nor $\mathscr{T}_{2}$ for $h<h_{\mathrm{c}}$, while it intercepts $\mathscr{T}_{1}$ and $\mathscr{T}_{2}$ for $h>h_{\mathrm{c}}$; second, $\mathscr{P}$ intercepts $\mathscr{T}_{1}$ for $h<h_{\mathrm{c}}$, while it intercepts $\mathscr{T}_{2}$ for $h$ $>h_{\mathrm{c}}$; third, $\mathscr{P}$ intercepts neither $\mathscr{T}_{1}$ nor $\mathscr{T}_{2}$ for $h>h_{\mathrm{c}}$, while it intercepts $\mathscr{T}_{1}$ and $\mathscr{T}_{2}$ for $h<h_{\mathrm{c}}$. We denote by $\alpha_{1}$ and $\alpha_{2}$ the angles of $\mathscr{T}_{1}$ and $\mathscr{T}_{2}$, respectively, with $\mathscr{P}$. In the first and third cases, for the values of $h$ for which $\mathscr{P}$ and $\mathscr{T}_{1}$ and $\mathscr{P}$ and $\mathscr{T}_{2}$ intersect, the section is a rectangle of length $A B$ and width $\left|\cot \left(\alpha_{1}\right)-\cot \left(\alpha_{2}\right)\right||\varepsilon|$ plus two parts which depend on the details of the shape around $\mathbf{A}$ and $\mathbf{B}$ but of which the areas vary quadratically with $\varepsilon$. Therefore, we have $\lambda=1$ and an asymptotic behaviour for the amplitude form factor in $A B\left|\cot \left(\alpha_{1}\right)-\cot \left(\alpha_{2}\right)\right| q^{-2}$. In the second case, for $h<h_{\mathrm{c}}$ a part of the section perimeter is a straight line of length $A B$ and of position varying as $h \cot \left(\alpha_{1}\right)$, while for $h>h_{\mathrm{c}}$ the same straight line has its position varying as $h \cot \left(\alpha_{2}\right)$. Therefore, a slope discontinuity exists for $A(h)$ equal to $A B\left[\cot \left(\alpha_{1}\right)-\cot \left(\alpha_{2}\right)\right]$. Again, we have $\lambda=1$ and an asymptotic behaviour for the amplitude form factor in $A B\left|\cot \left(\alpha_{1}\right)-\cot \left(\alpha_{2}\right)\right| q^{-2}$.

4.4. $\lambda=3 / 2$ : point contact with the curved edge of a planar truncation

We will now consider the case of an object having a planar truncation $\mathscr{T}$ with a curved edge, and we will study the singularity linked to the contact of $\mathscr{P}$ with this curved edge for $h=h_{\mathrm{c}}$. Two cases must be considered.

Firstly, the contact $\mathbf{t}$ with $\mathscr{T}$ is the first (or the last) contact of $\mathscr{P}$ with the body. We will denote as $\alpha$ the angle between $\mathscr{T}$ and $\mathscr{P}$ and as $\beta$ the angle between $\mathscr{P}$ and the plane tangent to the body in t. Therefore, the section for $h \lesssim h_{\mathrm{c}}$ is a circular segment of radius $r$ and of sagitta $f=\varepsilon[\cot (\alpha)+\cot (\beta)]$. Its area is

$$
\begin{aligned}
A(h) & =r^{2}\left\{\arccos (1-f / r)-(1-f / r)\left[1-(1-f / r)^{2}\right]^{1 / 2}\right\} \\
& =4\left(2^{1 / 2}\right) / 3 r^{1 / 2} f^{3 / 2}+\mathcal{O}\left(f^{5 / 2}\right) \\
& =4\left(2^{1 / 2}\right) / 3 r^{1 / 2} \varepsilon^{3 / 2}[\cot (\alpha)+\cot (\beta)]^{3 / 2}+\mathcal{O}\left(\varepsilon^{5 / 2}\right) .
\end{aligned}
$$

Therefore, the contact leads to a singularity with $\lambda=3 / 2$ and an asymptotic behaviour in $4 / 3\left(2^{1 / 2}\right) r^{1 / 2}[\cot (\alpha)+$ $\cot (\beta)]^{3 / 2} q^{-5 / 2}$. The details of the body shape determine the value of $r$. For a truncated sphere of radius $R$ truncated at a height $H=R \cos \left(\varphi_{0}\right)$, we have $r=R \sin \left(\varphi_{0}-\alpha\right)$ and $\beta=\varphi_{0}-\alpha$, leading to an asymptotic behaviour in $\left[4\left(2^{1 / 2}\right) R^{1 / 2} \sin \left(\varphi_{0}\right)^{3 / 2}\right] /$ $\left[3 \sin \left(\varphi_{0}-\alpha\right) \sin (\alpha)^{3 / 2}\right] q^{-5 / 2}$ (see Fig. 3). In this case, the condition for the contact between $\mathscr{T}$ and $\mathscr{P}$ being the first contact is $\alpha<\varphi_{0}$. For a right revolution cylinder of radius $R$ (see Fig. 2), we have $r=R \cos (\alpha)$ and $\beta=\pi / 2-\alpha$, leading to an asymptotic behaviour in $\left[4\left(2^{1 / 2}\right) R^{1 / 2}\right] /\left[3 \cos (\alpha) \sin (\alpha)^{3 / 2}\right] q^{-5 / 2}$, in full agreement with the asymptotic behaviour which can be deduced from the direct formula. In this case, the contact between $\mathscr{T}$ and $\mathscr{P}$ is the first for $0 \leq \alpha \leq \pi / 2$. 


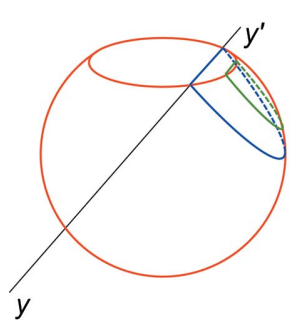

(a)

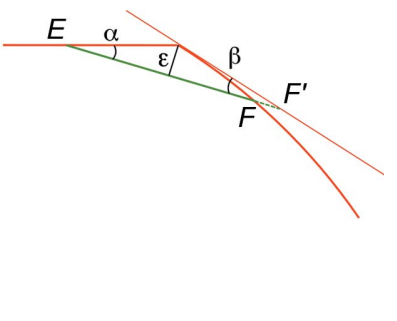

(b)
Figure 3

Sections of a truncated sphere. Red denotes the surface of the truncated sphere, and blue and green the sections by a generic plane for two different values of $h$. (a) Perspective view. (b) A view normal to $y y^{\prime}$. To first order in $\varepsilon, E F^{\prime}=E F=[\cot (\alpha)+\cot (\beta)] h$.

Secondly, the contact between $\mathscr{T}$ and $\mathscr{P}$ does not correspond to the entry or the exit. The singularity is essentially the same, but now the circular segment contributes like a difference between the shape before the contact and the shape after the contact, quite similar to the case studied for the tetrahedron. In this case, the rule of dominant singularities may apply, avoiding the observation of an asymptotic term in $q^{-5 / 2}$ associated with the circular segment. This is the case for the truncated sphere, for which the entry or exit by a spheroid part leads to an asymptotic behaviour in $q^{-2}$. But this is not the case for the cylinder for which, in a generic direction, we have four contacts with the bases' curved edges, all of them leading to an asymptotic behaviour in $q^{-5 / 2}$.

\section{5. $\lambda=2$ : contact with an apex}

We will show that this contact leads to $\lambda=2$ and an asymptotic term in $q^{-3}$. Since all the other singularities lead to smaller values of $\lambda$, the observation of this singularity implies that the contact with an apex should occur at both the entry and the exit of the body. For the sake of simplicity, we will limit our discussion to the entry case. In this case, for $h<h_{\mathrm{i}}$, the section is a polygon, with or without curvilinear parts, of shape given by the detailed shape of the apex. This polygon can be decomposed into several parts, namely a standard straight polygon and circular segments associated with each curvilinear side. The size of the straight polygon varies locally homothetically with $\varepsilon$. Therefore, its section area varies quadratically with $\varepsilon$. The sagitta of the circular segments associated with each curvilinear side varies quadratically with $\varepsilon$. Using the relation between the sagitta of a circular segment and its area established in $\$ 4.2$, we conclude that the circular segments associated with the curvilinear sides have areas varying as $\varepsilon^{3}$ (see Appendix $B$ for detailed calculations). Therefore, we have $\lambda=2$ for the whole section and an asymptotic behaviour in $q^{-3}$.

\subsection{On nesting}

It may often occur that, for a given direction, we meet two singularities of the same $\lambda$. This may correspond to two parallel faces; to the entry and exit by two parallel generatrices; to the entry and exit by two spheroidal points; to a $\mathbf{q}$ direction perpendicular to two edges; and finally to the fringe pattern parallel to the great circle perpendicular to an edge which corresponds to the nesting between the two apices terminating the edge (Croset, 2017). In all these cases, the asymptotic behaviour exhibits fringes of period $2 \pi /\left|h_{1}-h_{2}\right|$, the fringe contrast being dependent on the amplitude of the two singularities. We will not discuss such nesting cases in detail, since the purpose of this work is the discussion on a large scale of the contrast behaviour of intensity maps of $F(\mathbf{q})$. However, this nesting phenomenon is the price to pay for the observation of singularities of large $\lambda$, since both the rule of dominant singularities and the frequent centrosymmetry of the bodies lead to the study of bodies presenting nesting. An accurate description of the fringe pattern is quite complex since its details depend on numerous parameters: the quality of the 'parallelism' of two truncations, the equality or inequality of the amplitudes of the interfering singularities, Moiré patterns due to superpositions of fringe patterns occurring in different directions etc. All these features contain information which can lead, in a numerical approach, to quite fine details of the body shape, but we think that the fringe pattern is too complex to be addressed in a general analytical approach.

\section{An exhaustive study: the fourfold truncated sphere}

So far, we have studied analytically the asymptotic behaviours of $F(\mathbf{q})$ and we have shown that they can be related to the shape singularities of the body. In this section, we will discuss numerically the $q$ domain of validity of these predictions for a precise example, the fourfold truncated sphere. We will show that the contrast associated with the different asymptotic behaviours is observable for reasonable values of $q$, i.e. $q R=100$.

\subsection{Choice of the studied body}

We want to construct a rounded body that is as simple as possible while allowing the observation of the singularities associated with a face, a straight edge, a spheroidal part, a curved edge and an apex. Using two secant truncations on a sphere of radius $R$, we obtain all the required objects. In order to circumvent the rule of dominant singularities and to allow the observation of all the asymptotic behaviours, the simplest choice is to choose to build a centrosymmetric object, i.e. a fourfold truncated sphere. To simplify one step further, we choose to have the $z$ axis as a fourfold symmetry axis, leading to four truncations in $x=R \cos \left(\varphi_{0}\right), x=-R \cos \left(\varphi_{0}\right), y=$ $R \cos \left(\varphi_{0}\right)$ and $y=-R \cos \left(\varphi_{0}\right)$. We take $\varphi_{0}=\pi / 3$. Choosing the $z$ axis as the polar axis, we denote $\varphi$ the elevation angle and $\theta$ the azimuthal angle of $\mathbf{q}$. The symmetries of the chosen object, the $z$ axis as the fourfold symmetry axis and the sphere centre as the inversion centre, allow us to restrict the angular domain of study to $0 \leq \varphi \leq \pi / 2$ and $0 \leq \theta \leq \pi / 4$ and to restrict the study of the section and of $A(h)$ to positive values of $h$ for each q direction. 


\subsection{Classification of the q direction}

We will denote by $\mathscr{T}_{1}$ the truncation corresponding to $x=$ $R \cos \left(\varphi_{0}\right)$ and $\mathscr{T}_{2}$ the truncation corresponding to $y=$ $R \cos \left(\varphi_{0}\right), \mathbf{E}$ the straight edge corresponding to the intersection between $\mathscr{T}_{1}$ and $\mathscr{T}_{2}$, and $\mathbf{A}$ the extremity of $\mathbf{E}$ belonging to the angular domain studied. Let us study the nature of the entry points for the different $\mathbf{q}$ directions, indicated by their positions on the unit sphere.

(i) The direction $\varphi=0, \theta=0$ leads to $\mathbf{q}$ normal to the face $\mathscr{T}_{1}$. The entry point corresponds to the contact with the whole face. We will denote by $\mathbf{C}_{1}$ the point of the unit sphere corresponding to this direction.

(ii) The great circle $\varphi=0$ corresponds to $\mathbf{q}$ perpendicular to the edge $\mathbf{E}$. For the part of the great circle corresponding to $0<$ $\theta<\pi / 4$, the entry point corresponds to the contact with the edge $\mathbf{E}$.

(iii) For $\varphi>\arccos \left[\cos \left(\varphi_{0}\right) / \cos (\theta)\right]$, the entry point is the non-truncated part of the sphere. The boundary of this locus corresponds to the circular edge of the truncation of the unit sphere by the plane $z=\cos \left(\varphi_{0}\right)$. We refer to this circle as $\mathscr{C}_{\mathscr{T}_{1}}$.

(iv) For

$$
\varphi<\arccos \left[\frac{\cos \left(\varphi_{0}\right)^{2}}{\cos (\theta)^{2} \cos \left(\varphi_{0}\right)^{2}+\sin (\theta)^{2} \sin \left(\varphi_{0}\right)^{2}}\right]^{1 / 2},
$$

the entry point is $\mathbf{A}$, corresponding to $\mathbf{A}^{\prime}$ on the unit sphere. The boundary of this locus corresponds to the arc of the great circle joining $\mathbf{A}^{\prime}$ to the point $\mathbf{C}_{1}$. We will refer to this boundary as $\mathscr{C}_{\mathbf{A}^{\prime} \mathbf{C}_{1}}$.

(v) Inside the triangle delimited by the three circles $\mathscr{C}_{\mathscr{T}_{1}}$, $\mathscr{C}_{\mathbf{A}^{\prime} \mathbf{C}_{1}}$ and $\varphi=0$, the entry point corresponds to the contact with the circular edge of $\mathscr{T}_{1}$.

Using the symmetries of the body a full map of the different entry points can be drawn (Fig. 4).

In order to compute $F(\mathbf{q})$ we must find, for a given $\mathbf{q}$ direction, all the section singularities occurring in the interval $h_{\mathrm{o}} \leq h \leq h_{\mathrm{i}}$ and identify the section shape for each sub-interval

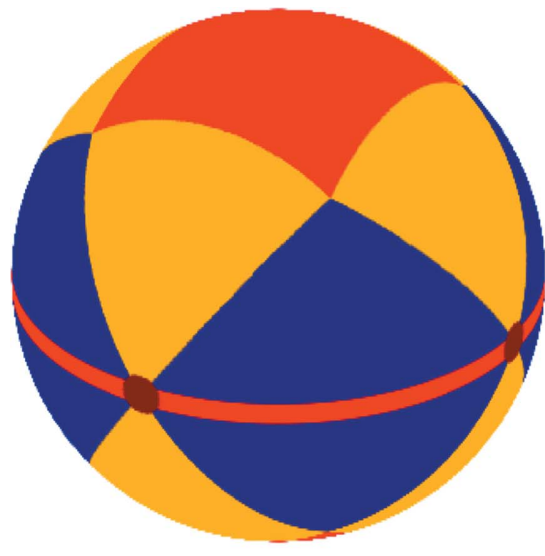

(a)

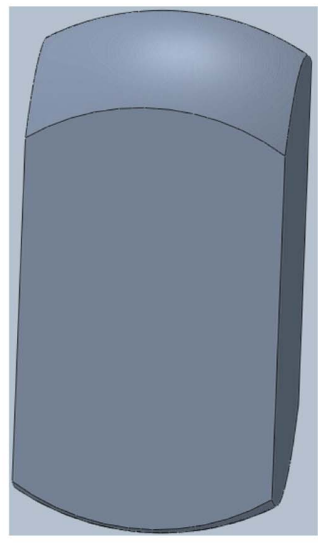

(b)
Figure 4

(a) A map of the $\lambda$ values for a fourfold truncated sphere. The poles $\varphi=0$ and $\theta=n \pi / 2$, and the circle $\varphi=0$, are widened for the sake of visibility. Brown denotes $\lambda=0$, red $\lambda=1$, orange $\lambda=3 / 2$ and blue $\lambda=2$. (b) A fourfold truncated sphere. $h_{n-1} \leq h \leq h_{n}$. Even for the very symmetric case we have chosen, such a task divides the angular domain $(0 \leq \varphi \leq \pi / 2$; $0 \leq \theta \leq \pi / 4)$ into 18 different regions, each of which leads to four to eight sub-intervals for $h$. These large numbers make the sections method rather tedious to implement numerically. Nevertheless, in the symmetric case that we have chosen, for every q direction, the exponent of the entry singularity is inferior or equal to all the critical exponents met in this direction. This implies that the map of Fig. 4 describes not only the different entry points but also the different asymptotic behaviours. It exhibits poles in the direction normal to the faces for which the asymptotic behaviour is in $q^{-1}$, two regions outside the truncations around the $z$ axis for which the asymptotic behaviour is the common $q^{-2}$ expected for spheroidal objects, a great circle joining the poles for which the asymptotic behaviour is again in $q^{-2}$, four curvilinear quadrilaterals around this great circle for which the asymptotic behaviour is in $q^{-3}$, and eight curvilinear triangles for which the asymptotic behaviour is in $q^{-5 / 2}$. Note that the presence of a strong singularity in the body shape is not only at the origin of an asymptotic trailing edge in the $\mathbf{q}$ direction 'perpendicular' to this singularity but also the cause of a decrease in the asymptotic behaviour in the surrounding region because of its boundary. In our case, a truncation leads to a pole in $q^{-1}$ and to a region in $q^{-5 / 2}$ due to its curved edge, while a straight edge leads to a circle in $q^{-2}$ and a region in $q^{-3}$ due to its two extremities. It is as though the excess intensity was locally pumped.

\subsection{The validity domain of the asymptotic predictions}

Fig. 5 shows the amplitude form factor modulus for $q R=$ $100 \simeq 16 \times 2 \pi$, together with the calculated boundaries of the different asymptotic regions. The contrast is in remarkable agreement with the map of the asymptotic behaviour. The

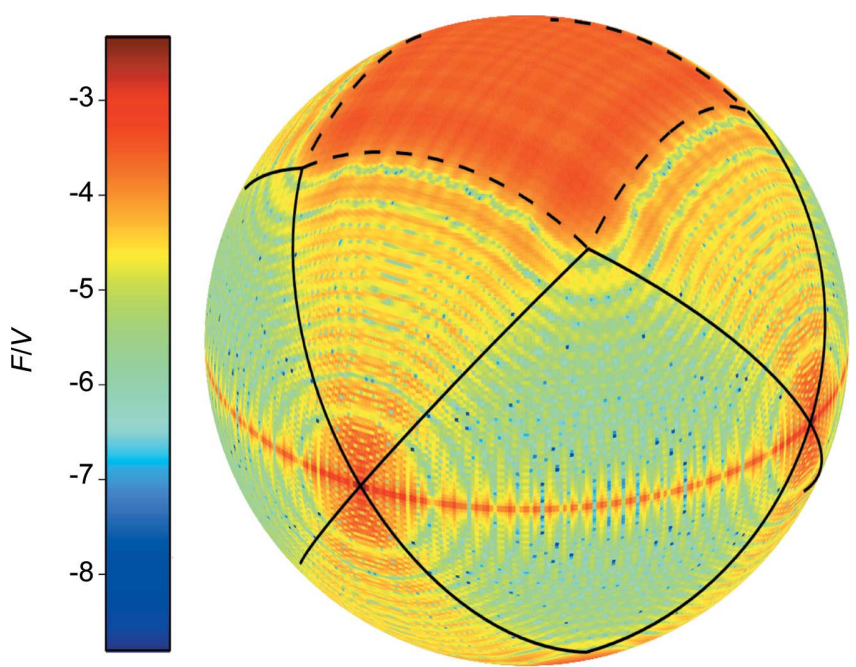

Figure 5

The amplitude form factor of a fourfold truncated sphere at $q R=100$. The colour scale is logarithmic. The form factor amplitude, $F$, is normalized by the body volume, $V$. Dashed lines denote the $\mathscr{C}_{\mathscr{T}_{1}}$ circular arc and its symmetry-equivalent arcs. Solid lines denote the $\mathscr{C}_{\mathbf{A}^{\prime} \mathbf{C}_{1}}$ circular arc and its symmetry-equivalent arcs. 
excess intensities associated with the $x$ and $y$ poles and the great circle joining them are clearly visible, together with three different regions: strong intensity around the $z$ axis, medium intensity in the triangles and low intensity in the curvilinear quadrilateral. Note that the boundaries between the different regions are clear and follow the analytical predictions very well.

\section{Conclusions}

The Patterson result, which shows that the amplitude form factor of a body can be understood as the Fourier transform of the sections of the body by planes normal to the scattering vector, has allowed us to establish a strong relationship between the singularities of the body surface and the asymptotic behaviour of the amplitude form factor at large $\mathbf{q}$. We have studied the contacts of an intersecting plane with the main possible singularities of the body surface. All the studied cases lead to an asymptotic behaviour in $q^{-(\lambda+1)}$, with $\lambda$ a half integer and $0 \leq \lambda \leq 2$. The strong singularities of the surface truncation, straight edge - are associated with both narrow regions of the orientation sphere, for which $\lambda$ is low, and surrounding extended regions, for which $\lambda$ is high. A numerical study shows that this analysis is pertinent to describing the intensity landscape for rather small values of $q: q R=100 \simeq$ $16 \times 2 \pi$ for a truncated sphere of radius $R$. Since, for the reconstruction of direct space, the reciprocal-space regions of both high and low scattering intensities are relevant, we think that this approach, the sections method, will be useful in studies of objects by coherent scattering with the new highbrightness X-ray sources. Since the presence of apices is strictly associated with $q^{-3}$ asymptotic behaviour, we believe that it is of primary importance to collect experimental data in the low counting rate regions.

\section{APPENDIX $A$}

\section{The cone case}

For the sake of simplicity, we will consider the case of a revolution cone, $\mathscr{C}_{t}$, truncated by two planes normal to its axis at height $z_{1}$ and $z_{2}$. We will denote by $\alpha$ the half apical angle. When the angle between $\mathscr{P}$ and the cone axis is equal to $\alpha$, the first contact between $\mathscr{P}$ and $\mathscr{C}_{t}$ is a portion of the directrix. For lower values of $h$, the section is a curvilinear trapezoid of which two sides are arcs of the same parabola. Choosing in the plane $\mathscr{P}$ the parabola directrix as the $Y$ axis and its normal passing through the focus as the $X$ axis, the parabola equation is $Y^{2}=2 \varepsilon \tan (\alpha) X$. The area of the section is written as

$$
S(\varepsilon)=2 \int_{x_{1}}^{x_{2}} Y \mathrm{~d} X
$$

and varies as $\varepsilon^{1 / 2}$. Thus, we have $\lambda=1 / 2$. Full calculations lead to

$$
S(\varepsilon)=4\left(2^{1 / 2}\right) / 3 \varepsilon^{1 / 2} \sin (\alpha)^{1 / 2} \cos (\alpha)^{-2}\left(z_{2}^{3 / 2}-z_{1}^{3 / 2}\right) .
$$

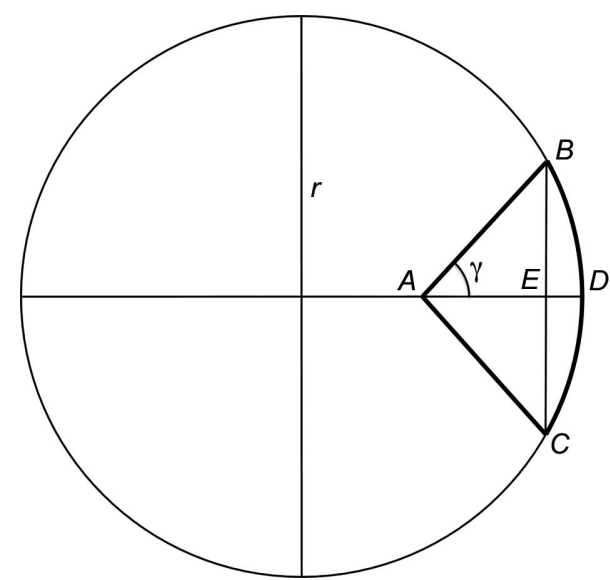

Figure 6

Curvilinear triangle.

\section{APPENDIX $B$}

\section{Curvilinear triangle}

Let us consider the curvilinear triangle $A B C$ drawn in Fig. 6. We will denote as $v$ the length $A D$, and we will seek the lowest term in $v$ of the area of the curvilinear triangle, $S(v)$. This area is the sum of the area of the straight triangle $A B C$ plus the area of the circular segment $B C$. The key point is that the sagitta of the circular segment, $E D$, is of second order in $v$ and that $A E=A D$ to first order in $v$. The area of the straight triangle is therefore equal to $v^{2} \tan (\gamma)$ to the lowest order in $v$ while, using the result obtained in $\S 4.4$, the area of the circular segment is of third order in $v$. The first term of the series development of the area of $S(v)$ is therefore $v^{2} \tan (\gamma)$.

\section{Acknowledgements}

The author thanks Olivier Thomas for pointing out the seminal work of Patterson, Denis Limagne for his precious help in preparing the figures and Ian Vickridge for careful reading of the manuscript.

\section{References}

Balmes, O., Prévot, G., Torrelles, X., Lundgren, E. \& Ferrer, S. (2016). ACS Catal. 6, 1285-1291.

Cho, K.-S., Talapin, D. V., Gaschler, W. \& Murray, C. B. (2005). J. Am. Chem. Soc. 127, 7140-7147.

Chourou, S. T., Sarje, A., Li, X. S., Chan, E. R. \& Hexemer, A. (2013). J. Appl. Cryst. 46, 1781-1795.

Coati, A., Creuze, J. \& Garreau, Y. (2005). Phys. Rev. B, 72, 115424. Croset, B. (2017). J. Appl. Cryst. 50, 1245-1255.

Diaz, A., Mocuta, C., Stangl, J., Mandl, B., David, C., Vila-Comamala, J., Chamard, V., Metzger, T. H. \& Bauer, G. (2009). Phys. Rev. B, 79, 125324.

Favre-Nicolin, V., Eymery, J., Koester, R. \& Gentile, P. (2009). Phys. Rev. B, 79, 195401.

Fournet, G. (1951). Bull. Soc. Fr. Miner. Cristallogr. 74, 39-113.

Georg, K. \& Tausch, J. (1994). Math. C, 62, 755-763.

Geuchies, J. J. et al. (2016). Nat. Mater. 15, 1248-1254.

Goubet, N., Albouy, P.-A., Thompson, A. \& Pileni, M.-P. (2016). CrystEngComm, 18, 6166-6175. 
Goubet, N., Yan, C., Polli, D., Portalès, H., Arfaoui, I., Cerullo, G. \& Pileni, M.-P. (2013). Nano Lett. 13, 504-508.

Gruner, M. E. G., Rollmann, G., Entel, P. \& Farle, M. (2008). Phys. Rev. Lett. 100, 087203.

Guelfand, I. M. \& Chilov, G. E. (1962). Les Distributions. Paris: Dunod.

Guinier, A. \& Fournet, G. (1955). Small-Angle Scattering of X-rays. New York: Wiley and Sons.

Jones, M. R., Macfarlane, R. J., Lee, B., Zhang, J., Young, K. L., Senesi, A. J. \& Mirkin, C. A. (2010). Nat. Mater. 9, 913-917.

Kalesaki, E., Delerue, C., Morais Smith, C., Beugeling, W., Allan, G. \& Vanmaekelbergh, D. (2014). Phys. Rev. X, 4, 011010.

Labat, S., Richard, M. I., Dupraz, M., Gailhanou, M., Beutier, G., Verdier, M., Mastropietro, F., Cornelius, T. W., Schülli, T. U., Eymery, J. \& Thomas, O. (2015). ACS Nano, 9, 9210-9216.

Langille, M. R., Personick, M. L., Zhang, J. \& Mirkin, C. A. (2012). J. Am. Chem. Soc. 134, 14542-14554.

Lazzari, R. (2002). J. Appl. Cryst. 35, 406-421.

Lee, J.-S., Kovalenko, M. V., Huang, J., Chung, D. S. \& Talapin, D. V. (2011). Nat. Nanotechnol. 6, 348-352.
Miao, J. W., Ishikawa, T., Robinson, I. K. \& Murnane, M. M. (2015). Science, 348, 530-535.

Murphy, C. J., Sau, T. K., Gole, A. M., Orendorff, C. J., Gao, J., Gou, L., Hunyadi, S. E. \& Li, T. (2005). J. Phys. Chem. B, 109, 1385713870.

Öztürk, H., Huang, X., Yan, H., Robinson, I. K., Noyan, I. C. \& Chu, Y. S. (2017). New J. Phys. 19, 103001.

Patterson, A. L. (1939). Phys. Rev. 56, 972-977.

Renaud, G., Lazzari, R. \& Leroy, F. (2009). Surf. Sci. Rep. 64, 255-380.

Robinson, I. K. (1986). Phys. Rev. B, 33, 3830-3836.

Senesi, A. \& Lee, B. (2015). J. Appl. Cryst. 48, 565-577.

Xia, Y., Xiong, Y., Lim, B. \& Skrabalak, S. E. (2009). Angew. Chem. Int. Ed. 48, 60-103.

Xie, S., Lu, N., Xie, Z., Wang, J., Kim, M. J. \& Xia, Y. (2012). Angew. Chem. Int. Ed. 51, 10266-10270.

Yamada, Y., Tsung, C.-K., Huang, W., Huo, Z., Habas, S. E., Soejima, T., Aliaga, C. E., Somorjai, G. A. \& Yang, P. (2011). Nat. Chem. 3, 372-376.

Zhang, H., Jin, M. \& Xia, Y. (2012). Angew. Chem. Int. Ed. 51, 76567673. 